\title{
Transnationale Verflechtungen und transgenerationale Folgen von Verfolgung, Flucht und Migration: Familien- und Lebensgeschichten von Roma aus Ex-Jugoslawien
}

\author{
Doreen Blume-Peiffer
}

\section{Einleitung}

Betrachtet man die historische Aufarbeitung der Verfolgungsgeschichte und des antifaschistischen Widerstandes von Roma in Jugoslawien während des Zweiten Weltkriegs, so verdeutlicht dies, dass Roma als Opfer des Nationalsozialismus, aber auch als antifaschistische Partisanenkämpfer*innen in der Geschichtsschreibung kaum Berücksichtigung fanden. Die Beteiligung von Roma am erfolgreichen Befreiungskampf der Tito-Partisan*innen findet sich in der Geschichtsschreibung nur als Randnotiz. Blicken wir auf die gewaltsamen Konflikte, die im Zuge des Zerfalls Jugoslawiens in den 1990er Jahren eskalierten, zeigen diese Kontinuitäten der Gewalt, die kollektivgeschichtlich nicht aufgearbeitet wurden. Am 12. Juli 1992 fand z.B. ein Massaker im bosnischen Dorf Skočić statt, bei dem 28 Roma, darunter auch Frauen und Kinder, ermordet wurden. Das Kriegsverbrechen wurde von einer TschetnikGruppierung namens Simini četnici begangen. Opfer, die diesen Angriff überlebt hatten, berichteten von massiver Gewalt (vgl. Zeidler 2017: 44).

Blume-Peiffer, D. (2020): Transnationale Verflechtungen und transgenerationale Folgen von Verfolgung, Flucht und Migration. Familien- und Lebensgeschichten von Roma aus Ex-Jugoslawien. In: Bahl, E./Becker, J. (eds.): Global Processes of Flight and Migration. The Explanatory Power of Case Studies / Globale Flucht-und Migrationsprozesse. Die Erklärungskraft von Fallstudien. Göttingen: Göttingen University Press, 239-254. bttps:/ / doi.org/10.17875/ gир2020-1323 
Vor diesem Hintergrund widmet sich dieser Beitrag den familien- und kollektivgeschichtlichen Erfahrungen von Roma aus dem ehemaligen Jugoslawien, die während der 1990er Jahre infolge des Zerfalls Jugoslawiens und der daran anschließenden kriegerischen Auseinandersetzungen nach Deutschland geflohen sind. Während im deutschen medialen Diskurs stereotypisierende, homogenisierende Bilder von „Armutsmigranten“ verbreitet wurden, zeigt meine ethnographische Studie, die auf familiengeschichtlichen und biographischen Interviews basiert, sehr unterschiedliche familien- und lebensgeschichtliche Verläufe mit verschiedenen Migrations- und Mobilitätsmustern. Zeigen sich innerhalb der Familien kumulative Diskriminierungserfahrungen infolge asylrechtlicher Restriktionen, die Familienangehörige verschiedener Generationen belasten, so wirken ebenfalls Gewalt- und Verlusterfahrungen der Vergangenheit in die Gegenwart. Um transgenerationale Folgen von Verfolgung, Flucht und Migration zu verstehen, ist es notwendig, die Familien- und Kollektivgeschichte über einen längeren Zeitraum in den Blick zu nehmen. In diesem Beitrag beschäftige ich mich mit den transgenerationalen Folgen der leidvollen Erfahrungen von Roma, verbunden mit den belastenden und häufig traumatisierenden Erlebnissen während des Zweiten Weltkriegs, der Jugoslawienkriege und der Flucht. Wie gestaltet sich der innerfamiliäre Dialog über Erfahrungen während des Zweiten Weltkriegs und über die Gewalt- und Fluchterfahrungen in den 1990er Jahren infolge des Zerfalls Jugoslawiens? Wie wirken asylrechtliche Restriktionen nach der Migration nach Deutschland auf die Positionierungen und Selbstverortungen von Roma? Ich werde aufzeigen, dass Zugehörigkeitskonstruktionen vor dem Hintergrund divergierender Leidenserfahrungen in der Vergangenheit aber auch in der Gegenwart ausgehandelt werden. In den von mir geführten Interviews wurde deutlich, welch traumatisierende Auswirkungen solche Gewalterfahrungen wie z.B. die pogromartige Vertreibung von Roma aus dem Kosovo 1999 hatten. Interviewpartner*innen berichteten vom Verlust ihrer Häuser, dem Tod naher Angehöriger und angedrohter oder erlebter Gewalt. Die Rekonstruktionen der Familienund Lebensgeschichten zeigen des Weiteren, dass asylrechtliche Restriktionen, verbunden mit einer unsicheren Bleibeperspektive und der Angst vor Abschiebung, die Gegenwartsperspektive der interviewten Roma sehr bestimmen und die sich wandelnden Zugehörigkeitskonstruktionen beeinflussen.

Im Rahmen meiner ethnographischen Forschung habe ich Roma in Deutschland und Serbien interviewt. Zwischen Juni 2017 und November 2019 habe ich biographisch-narrative Interviews mit Roma aus Serbien und Kosovo geführt (vgl. Rosenthal 1995, 1997, 2005; Schütze 1977, 1983). Das Sample besteht aus 17 Interviewpartner*innen, 10 Frauen und 7 Männer, mit denen ich inklusive Follow-upTerminen 29 Interviews geführt habe. Bereits der Erhebungs- und Auswertungsprozess des ersten Falls verdeutlichte die erforderliche transnationale Perspektive und damit verbunden die Notwendigkeit der Forschung in Serbien und Deutschland. Die ersten Interviews habe ich in Deutschland geführt und bin dann in die Herkunftsorte meiner Interviewpartner*innen nach Serbien gereist, um weitere Familienangehörige vor Ort zu interviewen. 
Innerhalb der Familienverbände habe ich Interviews mit Familienangehörigen in verschiedenen Generationen geführt. Die Durchführung biographisch-narrativer Interviews stellt insbesondere in der Umsetzung als Mehrgenerationenanalyse die methodische Herangehensweise dieser Studie dar. Ziel ist u.a. dabei, lebensgeschichtliche Erfahrungen mit historischen und familiengeschichtlichen Ereignissen im Auswertungsprozess zu verknüpfen (vgl. Radenbach/Rosenthal 2012; Rosenthal 2005). Die Mehrgenerationenanalyse ermöglicht: 1) die Einbettung der Biographien in den Kontext der Sozialgeschichte; 2) die Analyse von Prozessen sozialer (einschließlich kollektiver) Phänomene über einen längeren historischen Zeitraum; 3) die Biographie und die Familiengeschichte in den Kontext von Zugehörigkeitsgruppen, Organisationen etc. zu stellen und somit 4) eine Überwindung der Trennung von Makro-, Meso- und Mikroperspektive (Radenbach/Rosenthal 2012: 5).

Im Folgenden möchte ich zunächst auf die asylrechtlichen Diskurse in Verbindung mit Bleibe- und Etablierungschancen von Roma in Deutschland und auf kumulative Diskriminierung in Serbien und im Kosovo eingehen. Im Anschluss werde ich auf den innerfamiliären Dialog über familien- und kollektivgeschichtliche Erfahrungen während des Zweiten Weltkriegs und in den 1990er Jahren und auf die Rezeption Tito-Jugoslawiens eingehen und abschließend die wesentlichen Ergebnisse zusammenfassen.

\section{Kein sicherer Ort für Roma: Zwischen Verfolgung, Duldung und „freiwilliger Rückkehr“،}

Der Schwerpunkt meiner Forschung liegt auf der Untersuchung kollektiv- und lebensgeschichtlicher Erfahrungen von Roma, die in den 1990er Jahren infolge der Jugoslawienkriege nach Deutschland migriert sind, wobei ich mich auf die Herkunftskontexte Serbien und Kosovo konzentriere. Infolge der Jugoslawienkriege gab es große Fluchtbewegungen, die nicht in erster Linie nach Westeuropa führten, sondern in Flüchtlingslager der Balkanregion. Beispielsweise gibt es eine große Anzahl Vertriebener infolge des Kosovo-Krieges. 1999, nach dem Nato-Angriff und dem Rückzug der Serben aus dem Kosovo, kehrten circa 800.000 kosovarische Albaner zurück und vertrieben Roma und Serben. Schätzungen gehen von etwa 100.000 Vertriebenen aus (ERRC 2011:21). Viele von ihnen flohen nach Serbien und leben zum Teil bis heute noch als „Internally Displaced Persons“ in Flüchtlingslagern oder informellen Siedlungen. In diesem Zusammenhang gibt es Berichte von Menschenrechtsorganisationen, die auf gesundheitliche Langzeitschäden von Roma infolge der Errichtung von UN-Flüchtlingslagern in bleiverseuchten Gebieten verweisen (vgl. GfbV 2018).

Transnationale Migrationen und Binnenmigrationen sind mit Blick auf die Geschichte Südosteuropas kein neues Phänomen. Ende des 19. und zu Beginn des 20. Jahrhunderts wanderten geschätzt 1,5 Millionen Menschen aus Südosteuropa in die USA aus (Goeke 2007: 130). Ab den 1920er Jahren erfolgten Migrationen verstärkt 
nach Frankreich, Belgien, Deutschland und in die Niederlande (ebd.; vgl. Sundhausen 1999: 143). Migration stellte auch eine familiäre Arbeitsstrategie dar, z.B. als ,pecalba“ (,Lohnarbeiter in der Fremde“ oder „Wanderarbeiter"). So wurde die saisonale Migration einzelner oder mehrerer vorwiegend männlicher Familienangehöriger in eine andere Region zum Zwecke der Arbeit bei periodischer Rückkehr in den Herkunftsort bezeichnet (ebd.; Morokvašić 1987: 59). Infolge des GastarbeiterAbkommens zwischen Jugoslawien und Deutschland, das 1968 in Kraft trat und 1972 endete, migrierten bis 1973 etwa eine halbe Million Jugoslaw*innen nach Deutschland (Goeke 2007: 136; Baučić 1973: 62). Auch danach fanden infolge des Familiennachzugs Migrationen nach Deutschland statt. Mit dem Zerfall Jugoslawiens in den 1990er Jahren nahmen Migrationsbewegungen nach Deutschland zu. Häufig konnten die ankommenden Flüchtlinge auf transnationale Netzwerke zurückgreifen, da bereits Familienangehörige zu Zeiten des Anwerbeabkommens nach Deutschland migriert waren. Je nach Zeitpunkt der Migration und Herkunftsort/region haben die in den 1990er Jahren geflohenen Roma unterschiedliche Gewalt-, Verfolgungs- und Diskriminierungserfahrungen gemacht. Die Zwangsrekrutierungen in das Militär wie z.B. in die JVA (Jugoslawische Volksarmee) 1991 zum Einsatz im Kroatienkrieg lösten Fluchtbewegungen bereits Anfang der 1990er Jahre aus. Es folgte der Bosnienkrieg 1992 bis 1995. Aus dem Kosovo floh die Mehrheit der Roma Ende der 1990er Jahre infolge des Kosovo-Krieges. In Deutschland angekommen, erhielten Roma mehrheitlich eine „Duldung“. ${ }^{1}$ Die Nichtanerkennung des Flüchtlingsstatus, asylrechtliche Restriktionen und damit verbunden die Einschränkung von Grundrechten verstärkten Unsicherheitsgefühle. 2014 wurden die Balkanstaaten Bosnien-Herzegowina, Serbien und Mazedonien von der Bundesregierung als sichere Herkunftsländer eingestuft, 2015 folgten Kosovo, Albanien und Montenegro. Seitdem haben Asylsuchende aus diesen Staaten wenig bis keine Aussicht auf eine Bleibeperspektive in Deutschland.

Gibt es auch keine zuverlässigen Erhebungen zu den ethnischen Zugehörigkeiten der abgelehnten Asylbewerber*innen aus den Balkanstaaten, kann man dennoch davon ausgehen, dass viele Roma aus Serbien, dem Kosovo und Mazedonien von den Gesetzesverschärfungen betroffen waren und sind (Rosa Luxemburg Stiftung Südosteuropa 2016: 6). Mit dem Asylpaket I 2015 traten verschiedene Restriktionen in Kraft, die zu einer deutlichen Verschärfung des Asylrechts führten. Die Gesetzesänderungen bezogen sich u.a. darauf, dass Abschiebungen wieder unangekündigt durchgeführt werden können und die Möglichkeiten der Leistungskürzungen erweitert wurden, z.B. wenn keine Identitätspapiere vorliegen. Mit dem Inkrafttreten des Asylpakets II 2016 sind neben den beschleunigten Asylverfahren und der Unterbringung in „Ankunfts- und Rückführungseinrichtungen“ auch Veränderungen bezüglich des Schutzes kranker und traumatisierter Menschen zu vermerken. Nur noch schwere oder lebensbedrohliche Erkrankungen können eine Abschiebung abwen-

${ }^{1}$ Vgl. dazu \60a AufenthG. 
den, psychische Erkrankungen werden in dem Gesetzespaket nicht als „schwerwiegende Krankheit“ definiert (Bundesgesetzblatt 2016).

Die erwähnten schwierigen und häufig traumatisierenden Erlebnisse sind als Fluchtgründe von Roma kein Gegenstand des öffentlichen Diskurses, vielmehr überwiegen in der medialen Darstellung stereotypisierende Bilder. Die Gesetzesverschärfungen haben nicht nur Auswirkungen auf die ab 2015 nach Deutschland eingewanderten Roma, sondern auch auf jene Familienangehörige, die bereits seit den 1990er Jahren nach Deutschland geflohen waren und ihren Aufenthalt im Zuge der Altfallregelung, ${ }^{2}$ einer stichtagsabhängigen Bleiberechtsregelung für langjährig Geduldete, nicht verfestigen konnten. Wobei beide Gruppen häufig identisch sind, da, wie in den Fallrekonstruktionen deutlich wird, auch viele der ab 2015 nach Deutschland migrierten Roma bereits in den 1990er Jahren in Deutschland gewesen waren und aufgrund des prekären Aufenthaltsstatus zurückgehen mussten oder „freiwillig“ zurückkehrten. Die Deklarierung verschiedener Balkanländer wie Serbien und Kosovo als sichere Herkunftsstaaten in Deutschland stellt eine strukturelle Benachteiligung für marginalisierte Gruppen wie Roma dar. Die Mehrheit der Roma in Serbien lebt in informellen Siedlungen mit sehr niedrigem Lebensstandard und schlechter medizinischer Versorgung (vgl. Vuković 2016: 22). Auch die Situation der Roma im Kosovo ist prekär. Benachteiligungen im Bildungssektor, sehr hohe Arbeitslosigkeit, Diskriminierung beim Zugang zum Gesundheitswesen, racial profiling, hate crimes etc. haben zu einem signifikanten Rückgang der Roma-Bevölkerung im Kosovo geführt (ERRC 2011: 47 f.). Die Marginalisierung von Roma stellt in Serbien, im Kosovo aber auch in Deutschland und ganz Europa ein verbreitetes Problem dar und ist verbunden mit wirtschaftlicher und sozialer Ausgrenzung. Das Gefühl der Nichtzugehörigkeit zur Mehrheitsgesellschaft wird durch diese kumulativen Diskriminierungserfahrungen gestärkt und zeigt sich in vielfältigen Problemlagen. In Deutschland wirken asylgesetzliche Restriktionen Bleibe- und Etablierungsmöglichkeiten einer gesellschaftlichen Teilhabe entgegen. „Freiwillig“ Zurückgekehrte oder abgeschobene Roma haben wiederum in Serbien oder Kosovo eine Außenseiterposition inne.

Familien von Roma, die infolge des Zerfalls Jugoslawiens nach Deutschland geflohen sind, teilen die kollektive Erfahrung einer langjährig unsicheren Bleibeperspektive in Deutschland. So konnten zwar einige Roma, die in dieser Phase nach Deutschland migriert sind, ihren Aufenthalt mittlerweile festigen, es gibt jedoch auch zahlreiche Fälle von langjährig Geduldeten. In meinem Sample zeigt sich, dass die Aufenthaltstitel innerhalb der Familien verschieden sind. Das bedeutet, dass in allen Familienverbänden das Thema Aufenthalt sehr bedeutsam ist bzw. dass die Angst besteht, dass einzelne Familienangehörige abgeschoben werden oder „freiwillig“

\footnotetext{
2 Vgl. \\ 104a und b AufenthG: „Einem geduldeten Ausländer soll abweichend von \5 Abs. 2 eine Aufenthaltserlaubnis erteilt werden, wenn er sich am 1. Juli 2007 seit mindestens acht Jahren oder, falls er zusammen mit einem oder mehreren minderjährigen ledigen Kindern in häuslicher Gemeinschaft lebt, seit mindestens sechs Jahren ununterbrochen geduldet, gestattet oder mit einer Aufenthaltserlaubnis aus humanitären Gründen im Bundesgebiet aufgehalten hat ..." (Ausländerrecht 2008: 92).
} 
zurückkehren müssen. Das bedeutet aber zudem, dass die Etablierungschancen auch innerhalb der Familien unterschiedlich sind. Zudem wird sichtbar, dass Frauen und Kinder häufig den unsichersten Aufenthaltsstatus haben. Als Beispiel eines besonders prekären Falles verweise ich auf die Erfahrung einer 27-jährigen Romni, die 1993 in einer Kleinstadt in Nordrhein-Westfalen in Deutschland geboren wurde und seit ihrer Geburt in Duldung lebt. Auch zwei ihrer ebenfalls in Deutschland geborenen Kinder haben einen Duldungsstatus.

\section{Kollektivgeschichtliche Erfahrungen und transgenerationale Traumatisierung}

Empirische Untersuchungen zu Kriegsfolgen im ehemaligen Jugoslawien stehen derzeit noch am Anfang. Es wird dennoch deutlich, dass diese mit Verlust- und Gewalterfahrungen wie dem Tod naher Angehöriger, dem Verlust von sowohl physischer und psychischer Unversehrtheit als auch von Eigentum, Sicherheit und Vertrauen (Sundhausen 2014: 418) zusammenhängen:

Da viele Personen in wechselnden Konstellationen sowohl Opfer wie Täter waren, ergibt sich ein kompliziertes Gemisch aus teils traumatisierten, teils heroisierten, teils viktimisierten Erfahrungen, jeweils eingebunden in unterschiedliche politische, nationale und lokale Kontexte. (Sundhausen 2014: 417)

In dem Forschungsfeld der transgenerationalen Weitergabe oder auch Transmission von Traumata existiert eine Vielzahl an Forschungsarbeiten. Ich beziehe mich im Wesentlichen auf Hans Keilson (1979), der jüdische Kriegswaisen in den Niederlanden untersuchte, deren Kriegspflegefamilien sozio-ökonomisch, religiös und kulturell unterschiedlichen Milieus angehörten. Keilson arbeitete den prozessualen, sequentiellen Verlauf einer Traumatisierung heraus: 1) die Phase sozialer Stigmatisierung: Besetzung der Niederlande und anfängliche Verfolgung; 2) die Phase akuter Traumatisierung: Zeit der direkten Verfolgung und Deportation und 3) die Phase der Rehabilitation: Zeit nach dem Krieg, das Leben bei Angehörigen, in Waisenhäusern oder Pflegefamilien. Keilson analysierte Traumatisierungen in Korrelation mit dem jeweiligen Alter der Kinder, die Effekte einer kumulativ-massiven Traumatisierung und die Möglichkeiten und Wirkungen sozialer Rehabilitationen (ebd.: 12). Er kam zu dem Ergebnis, dass der Verlauf der dritten Phase, die Phase der Rehabilitation, entscheidend die Möglichkeiten der Traumabearbeitung beeinflusst (ebd.: 430).

Keilson folgend verweist David Becker (2014), aufbauend auf seinen psychotherapeutischen Arbeiten mit Folteropfern in Chile, auf den Zusammenhang individualpsychologischer und sozialpolitischer Traumatisierung. Becker kritisiert symptomargumentierende Traumakonzepte: „Wenn Traumata Teil eines gesellschaftspolitischen Prozesses sind, dann können sie nicht in die Sprache von Krankheitsdefinitionen hineingepresst werden, die diesen Prozessen gegenüberstehen und angeblich mit ihnen nichts zu tun haben." (Becker 2014: 70) Traumatisierungen müssen Becker 
zufolge im Kontext des politischen Prozesses Betrachtung finden, da sie sich auf gesamtgesellschaftliche Prozesse und Dynamiken beziehen. Die individualpsychologische Ebene wird ausschließlich in der Kontextualisierung der sozialpolitischen Ebene bearbeitbar (ebd.: 9).

Wie die Mehrgenerationsstudien von Gabriele Rosenthal und ihren Mitarbeiter*innen (1997) über den familiären Dialog in Familien von Überlebenden und Tätern der Shoah aufzeigten, fördern innerfamiliäres Schweigen und das Nichtwissen über die familiäre Vergangenheit die Weitergabe von Traumatisierungen: „Je geschlossener oder verdeckter der Dialog in der Familie ist, je mehr verheimlicht oder retuschiert wird, desto nachhaltiger wirkt sich die Familienvergangenheit auf die Kinder- und Enkelgeneration aus“ (Rosenthal 1997: 22). Das „Verschwiegene“ wird auf der latenten Kommunikationsebene z.B. über bestimmte Handlungsweisen weitergegeben. Zugleich können nachkommende Generationen Phantasien bezüglich der nicht-erzählten Inhalte der familien- und kollektivgeschichtlichen Vergangenheit entwickeln (vgl. Rosenthal 2001). Diesen Überlegungen folgend richte ich bei der Analyse transgenerationaler Folgen von Verfolgung, Flucht und Migration den Blick auf den innerfamiliären Dialog über familien- und kollektivgeschichtliche Erfahrungen. Ich schließe mich Keilsons und Beckers prozessualem Traumaverständnis an, dem Konzept der Sequentiellen Traumatisierung, das, wie Becker hervorhebt, nicht auf Krankheitssymptome reduziert ist, sondern die sozialpolitische Dimension von Traumatisierungen miteinschließt.

\subsection{Zwischen Verfolgung und aktivem Widerstand: \\ Roma im faschistisch besetzten Jugoslawien}

Die Kriegssituation während des Zweiten Weltkriegs war in Jugoslawien sehr unübersichtlich. Ethnische Spannungen brachen aus, die mit dem Zusammenschluss von ehemaligen Kriegsgegnern wie dem einst zu Österreich-Ungarn gehörenden Kroatien und dem zuvor unabhängigen Königreich Serbien im 1918 gegründeten Jugoslawien in Verbindung gebracht werden können. Noch vor dem Beginn des Zweiten Weltkriegs gründeten sich Tschetniks, serbische radikal-nationalistische Verbände, die für ein großserbisches Königreich kämpften, und die faschistische Ustascha-Bewegung, die ein katholisch und ethnisch reines Kroatien erkämpfen wollte. 1934 formierte sich die kommunistische Partei unter Josip Broz „Tito“, die die Gründung eines kommunistischen Vielvölkerstaates anstrebte. Nach dem Überfall der deutschen Wehrmacht auf Jugoslawien im April 1941 gelangte in Kroatien bereits wenige Tage später die faschistische Ustascha-Bewegung unter Ante Pavelić an die Macht und verfolgte Serben, Juden und Roma.

General Franz Böhme ordnete am 10. Oktober 1941 die Exekution von Geiseln als Vergeltungsmaßnahmen für die Tötung deutscher Wehrmachtsoldaten an. Bereits wenige Tage später fand im serbischen Kragujevac am 21. Oktober 1941 eine Massenexekution statt, bei der 2.300 Menschen, darunter zahlreiche Roma, erschossen wurden (Pisarri 2014: 148). Im Dezember 1941 wurde als Reaktion auf die Er- 
mordung von drei Wehrmachtsoldaten die Exekution von 300 Geiseln in Leskovac (Südserbien) von der nationalsozialistischen Besatzungsmacht angewiesen. Die Auswahl der Geiseln wurde den Stadtbeamten von Leskovac überlassen. Diese wählten daraufhin Bewohner*innen des Stadviertels Arapova Dolina aus, einer Gegend, in der mehrheitlich Roma lebten. Am Fuße des Berges Hisar wurden infolgedessen 293 Roma, 6 Juden und 11 Serben erschossen (Lawler 2017: 17; vgl. Pisarri 2014: 153). Mehr als 70 Konzentrationslager existierten während des Zweiten Weltkrieges in Jugoslawien, 329 Untersuchungsgefängnisse und darüber hinaus eine große Anzahl von Arbeitslagern (Djuric/Becken/Bengsch 1996: 277). Die Massendeportation von Roma, Juden und Serben in das KZ Jasenovac in Kroatien begann ab dem 19. Mai 1942. Auf Befehl des Reichsführers-SS Heinrich Himmler vom 16. Dezember 1942 sollten alle Sinti und Roma in das KZ Auschwitz-Birkenau deportiert werden. Ab dem Frühjahr 1943 erfolgten zahlreiche Deportationen nach Auschwitz auch aus Jugoslawien (Fings/Lissner/Sparing 1992: 52).

Die kollektivgeschichtlichen Erfahrungen von Roma aus Ex-Jugoslawien während des Zweiten Weltkriegs sind vielschichtig, Roma waren Opfer von Deportationen und Exekutionen, einige konnten sich in Wäldern verstecken und/oder schlossen sich den Partisanen an. Nur wenige Studien haben sich bis jetzt dezidiert mit dem Thema Widerstand von Roma während des Zweiten Weltkriegs in Jugoslawien befasst. Danijel Vojak (2017) widmete sich in „Roma Also Fought“ dem Thema der Partizipation von Roma in der antifaschistischen Partisanenbewegung in Kroatien und thematisierte die Nichtbeachtung dieser Involvierung in der Nachkriegszeit. Roma blieben als Opfer und auch als antifaschistische Partisanenkämpfer lange Zeit unsichtbar.

Die familien- und kollektivgeschichtlichen Erfahrungen von Roma während des Zweiten Weltkriegs wurden entsprechend meiner empirischen Befunde in den meisten Fällen nicht im Familiendialog bearbeitet. Dies zeigt sich im Nichtwissen oder der nur geringen Kenntnis der nachkommenden Generationen von den Erlebnissen ihrer Vorfahren während des Zweiten Weltkriegs. Innerfamiliäres Schweigen zu diesen belastenden Themen und Erfahrungen, aber auch ein Habitus des „Nicht-Fragens" von Kindern der Nachkriegsgeneration wurden in den Interviews als Gründe angegeben.

Zeigt sich auf Basis der empirischen Erhebung mehrheitlich wenig bis kein Wissen in den nachkommenden Generationen über die Erfahrung der Vorfahren während des Zweiten Weltkriegs, repräsentiert der Fall der Familie Markovic ${ }^{3}$ diesem entgegenlaufend die Weitergabe des Wissens, dass Vorfahren als Tito-Partisanen gekämpft haben. Rocko Markovic (1980 in Prstina geboren):

Ja, mein Opa war ein Partisan und sein Grab ist in Belgrad er ist da wo die sind alle Partisanen er ist da sein Name steht auch drauf in Belgrad da wo Tito ist //D: Ja// ... und mein Opa hatte so viele Orden er war ein guter Kämpfer er hat gut gekämpft.

${ }^{3}$ Alle Namen sind im Folgenden anonymisiert. 
In diesem Fall zeigt sich die Weitergabe des Wissens, dass Vorfahren als Tito-Partisanen gekämpft haben. Als Beleg wird auf das Grab des Großvaters in Belgrad auf dem Partisanenfriedhof verwiesen. Das Grab ist sehr bedeutsam für die Erinnerung und Tradierung. Jedoch wird nicht der Genozid an den Roma thematisiert, Roma werden nicht als Opfer dargestellt, sondern die kämpferischen Leistungen des Großvaters betont: „, hatte so viele Orden“; ,,er war gute Kämpfer er hat gut gekämpft" hier deutet sich eine Heroisierung der kämpferischen Leistungen des Großvaters an. Diese steht im Zusammenhang mit dem Grab auf dem Partisanenfriedhof und der Anerkennung in Form einer Hinterbliebenenrente zur Zeit Tito-Jugoslawiens. Im Fall Markovic wird der innerfamiliäre Dialog über die familien- und kollektivgeschichtlichen Erfahrungen der Vorfahren sichtbar und die Etablierung der Familie in der Vergangenheit und Gegenwart deutlich im Interview dargestellt. Rocko Markovic präsentierte seine Lebensgeschichte als Leidens- aber auch Erfolgsgeschichte. Als Leiden thematisiert er die Flucht 1999 aus dem Kosovo und als Erfolg stellt er heraus, dass er in der Gegenwart einen unbefristeten Arbeitsvertrag bekommen und sich sein Bleiberecht erfolgreich erkämpft habe. Rocko Markovic und alle seine Geschwister haben die deutsche Staatsbürgerschaft und stellen damit im Gesamtsample die in Deutschland etablierteste Familie dar.

Der Fall der Familie Mihaijlovic/Petrovic, einer serbisch-orthodoxen RomaFamilie, in der ich Interviews in drei Generationen, also auch mit einer Angehörigen der ersten Generation führen konnte, Julka Mihaijlovic (1935-2018), zeigt, dass das Wissen innerhalb des Familienverbandes unterschiedlich ist. Besteht in der zweiten Generation fragmentarisches Wissen, so zeigt sich in der dritten Generation wenig bis kein Wissen über die Erfahrungen der Vorfahren während des Zweiten Weltkriegs. Wie das Interview mit Julka Mihaijlovic sichtbar machte, schlossen sich die Vorfahren auch in diesem Fall kollektiv den Tito-Partisan*innen an, jedoch gab es kein Grab, keine Hinterbliebenenrente und keine Weitergabe dieser Involvierung an die folgenden Generationen. Das Interview mit Julka Mihaijlovic habe ich mit ihrer Enkelin Aleksandra Petrovic als Übersetzerin geführt. Die Enkelin erfuhr während des Interviews zum ersten Mal, dass ihr Urgroßvater als Tito-Partisan gekämpft hatte. Julka Mihaijlovic, die in ihrer Kindheit den Zweiten Weltkrieg erlebte, konnte die kollektivgeschichtlichen Erfahrungen nur bruchstückhaft zur Sprache bringen. Eine Familienangehörige der Linie Petrovic, Sarah Petrovic (1968 in Niś geboren), war die einzige Interviewpartnerin in dieser Familie, die den innerfamiliären Dialog über die Gewalterfahrungen während des Zweiten Weltkrieges andeutete. Sarah Petrovic:

Vater hat erzählt wie die sich versteckt haben und wie die die Häuser verbrannt haben wie sie von Dorf zu Dorf gezogen sind und wie sie sich verstecken mussten die ganze Zeit die Frauen vergewaltigt worden sind ... in dem Ort wo sie gewohnt haben ... da haben ja Tschetniks gelebt.

Während Sarah Petrovic belastende Themen wie die Vergewaltigungen ansprach und insbesondere Narrative über die Gewalt der Tschetniks im Herkunftsort ihrer 
Vorfahren darstellte, konnte Julka Mihaijlovic im Interview die in ihrer Kindheit erlebten Kriegs- und Verlusterfahrungen nur bruchstückhaft zur Sprache bringen.

\section{2 „Jeder war ein Jugoslawe“: Situation von Roma zur Zeit Tito-Jugoslawiens}

Die Proklamation Titos, unterschiedliche Gruppierungen zu vereinen, eröffnete Minderheiten wie Roma mehr Etablierungsmöglichkeiten (Bildungszugang, Arbeit, Gründung von Roma-Organisationen, etc.) als vor der Gründung oder nach dem Zerfall Jugoslawiens. Im damaligen Jugoslawien gab es verschiedene Kategorien: ethnische Gruppen, Nationalitäten (narodnosti), Nationen (narod), die wiederum im jugoslawischen Staat unterschiedliche Rechte hatten. In Jugoslawien kämpften Roma um die Anerkennung als Nationalität. Auch die Gründung zahlreicher RomaVereine in Jugoslawien stand mit diesem Anerkennungsbestreben in Verbindung. So gab es in den 1970er Jahren bereits über 60 Roma-Organisationen in Jugoslawien, die auch einen bedeutsamen Einfluss auf die weltweite Organisation von Roma hatten. 1971 fand der erste Welt-Roma-Kongress in London statt, auf dem der Oberbegriff „Roma“ “ für die Vielfalt existenter romanessprachiger Subgruppen festgesetzt sowie das Lied „Gelem Gelem“ als Nationalhymne festgelegt und eine eigene Flagge entworfen wurde (vgl. Hancock 2002: 120 f.).

In den von mir erhobenen biographisch-narrativen Interviews wurde die Zeit Tito-Jugoslawiens von der Generation der Nachkriegskinder als positiv herausgestellt, insbesondere wegen des kollektiven Wir-Gefühls: „Jeder war ein Jugoslawe.“ Familienangehörige der Nachkriegsgeneration, die in den 1950er und 1960er Jahren geborenen Roma, präsentierten ihre Lebenserfahrungen während der Zeit des Sozialismus als positiv, wobei insbesondere Tito glorifiziert und das kollektive WirGefühl erinnert wird. Gab es die übergeordnete gemeinsame Bezugskategorie „Jugoslawe", mit der sich zu Jugoslawienzeiten unterschiedliche Gruppen identifizierten, so verschwand diese gemeinsame Bezugskategorie mit dem Zerfall Jugoslawiens. In diesem Prozess löste sich nicht nur das Herkunftsland „Jugoslawien“ auf, sondern auch das kollektive Wir „Jugoslawe“. Aus der Gegenwartsperspektive wurde der Tod Titos 1980 von Interviewpartnern als Zäsur gedeutet. Sedat Jemini (1963 in Przren geboren): „Seit Tito gestorben ist ganze Welt ist kaputt ..." Als positiv herausgestellt wurde aus der Gegenwartsperspektive vor allem das kollektive Wir-Gefühl, das Gefühl der Sicherheit, Arbeit, Bildung und gegenseitiger Respekt. Dzoni Sichelschmidt, Gründer von Nevo Drom e.V., verweist in seinem Artikel „Wie meine Verfolgung begann“ (2015) auf eben dieses Gefühl der Nichtzugehörigkeit nach dem Zerfall Jugoslawiens:

Ich bin in einem Land aufgewachsen, das es nicht mehr gibt. Ich war Jugoslawe. Wir waren Jugoslawen. Meine Familie, unsere Verwandten - nicht Serben, Kroaten oder Albaner, das waren wir auch; vor allem aber Jugoslawen. Manchmal denke ich, wir Roma waren die einzigen Jugoslawen Jugoslawiens. 


\subsection{Zerfall Jugoslawiens: Zwangsrekrutierung, Verfolgung und}

Flucht/Migration: „Wir haben kein Land wir ham gar keine Heimat“

Die Involvierung und Betroffenheit von Roma während der Konflikte der 1990er Jahre ist vielschichtig und wird häufig als „zwischen den Fronten“ dargestellt. Meine empirische Studie zeigt, dass unterschiedliche Kriegserfahrungen innerhalb der Familien bestehen, je nach Zeitpunkt der Flucht und der Herkunftsregion vor der Migration. Im Zuge der Vertreibungen von Roma aus dem Kosovo flohen viele Roma Ende der 1990er Jahre aus der Region, wobei es auch Familien und/oder Familienangehörige gab, die bereits Anfang der 1990er nach Deutschland kamen, zurück nach Serbien oder Kosovo gingen oder deportiert wurden und später wieder nach Deutschland migrierten. Der Fall der Familie Jemini (Przren) sei hier als Beispiel angeführt. Die Familie migrierte, nachdem sie 1992 in Deutschland Zuflucht gesucht hatte, bereits ein Jahr später zurück in den Kosovo, und Familienangehörige erlitten im Zuge des Kosovokrieges 1998/1999 schwere physische und psychische Belastungen, wie die Vergewaltigung der Mutter in Anwesenheit der Kinder und des Ehemannes.

Familienangehörige von Roma, die 1999 aus dem Kosovo vertrieben wurden, teilen kollektive Vertreibungs- und Gewalterfahrungen. Im Sample berichteten alle Roma aus dem Kosovo vom Verlust ihrer Häuser, dem Verlust naher Angehöriger, von erlittener oder angedrohter sexueller Gewalt und Flucht. Interviewpartner*innen, die dies erlebt haben, begannen überwiegend das Interview mit der Kriegs/Fluchterfahrung, ohne auf die Zeit davor einzugehen. Der Fall der Familie Jemini zeigt exemplarisch auf, wie schwerwiegend die Gewalt- und Vertreibungserfahrungen von Roma Ende der 1990er Jahre im Kosovo waren: Im September 1999 wurde die Familie nachts von maskierten albanischen Nachbarn überfallen. Der damals vierjährige Sohn Milan Jemini erlebte, wie sein Vater Sedat mit einer Eisenstange geschlagen und mit einem Messer verletzt und seine Mutter in Anwesenheit der Familie vergewaltigt wurde.

Diese Gewalterfahrungen sowie die darauffolgende mehrmonatige Flucht durch die Wälder war zentrales Thema im Interview mit Sedat. Bei dem Interview mit Sedat Jemini (1963 in Przren geboren) war sein Sohn Milan Jemini (1995 in Przren geboren) anwesend. Dieser übersetzte aber erzählte auch aus seiner Perspektive, insbesondere dann, wenn sein Vater keine Worte für das Erlebte fand. Sedat Jemini begann nach der Eingangsfrage nach seiner Lebensgeschichte das Interview mit der Gewalterfahrung 1999 im Kosovo. Im weiteren Interviewverlauf wiederholte er mehrmals diese Situation, erzählte jedoch nur fragmentarisch und zeigte die physischen Narben, die er erlitten hatte. Dieser Fall repräsentiert Wissen und innerfamiliären Dialog über die kollektiv erlittenen Gewalterfahrungen in den 1990ern. Milan Jemini deutet: „Alle Kinder wissen, weil die es auch gesehen haben.“ Bis heute hat Sedat Jemini keinen gesicherten Aufenthalt in Deutschland, was die Phase der Rehabilitation nach erlittener Traumatisierung negativ beeinflusst. Die psychischen Folgen der Gewalt- und Vertreibungserfahrung werden durch das Gefühl der 
Unsicherheit infolge der fehlenden Bleibeperspektive in Deutschland gestärkt. Die Gewalterfahrungen in den 1990er Jahren, das kollektive Gefühl der Nichtzugehörigkeit nach dem Zerfall Jugoslawiens, die schwierige Phase der Rehabilitation nach der Ankunft in Deutschland zeigen sich als sequentielle Traumatisierung und wirken auf die Zugehörigkeitskonstruktionen in der Gegenwart. Im Sample zeigen sich unterschiedliche biographische Verläufe und Verortungen, allen gemeinsam ist jedoch Transkulturalität, verbunden mit Mehrfachverortungen und kulturellen Hybridisierungen (vgl. Nederveen Pieterse 2005; Bhabha 2000). Der Fall Jemini repräsentiert eine starke Vergangenheitsorientierung. Sedat Jemini verortet sich als Roma, er bezeichnet sich aber auch als Jugoslawe und stellt die Lebensumstände zur Zeit TitoJugoslawiens heraus: dass er Arbeit hatte, dass das soziale Miteinander positiver war, man sich sicher fühlte und keine Angst zu haben brauchte. Bis heute hat Sedat Jemini keinen gesicherten Aufenthalt in Deutschland. Wenn der sichere Ort in der Gegenwart fehlt und für die Zukunft ungewiss ist, sucht man ihn in der Vergangenheit. In dem Interview mit Sedat Jemini bestand eine starke Orientierung an dem kollektivem Wir „Jugoslawe“ in der Vergangenheit. Das Gefühl der Heimatlosigkeit zeigt sich auch in der nachfolgenden Generation, gerade vor dem Hintergrund der Gewalterfahrungen in den 1990er Jahren und der Marginalisierungserfahrung und kumulativen Diskriminierung in der Gegenwart. Milan Jemini:

Wir haben kein Land wir ham gar keine Heimat deswegen versteh ich auch nicht, dass manche Länder sagen ihr müsst in eure Heimat zurück aber Albaner im Kosovo sagen das ist doch gar nicht unsere Heimat. ... aber wo ist denn unsere Heimat eigentlich das frag ich mich oft und das ist das Problem.

Das Gefühl der Nichtzugehörigkeit zur Mehrheitsgesellschaft und der Verlust der Heimat stehen in Verbindung mit der marginalisierten Position von Roma im Kosovo, in Serbien, Deutschland und ganz Europa. „Wir haben kein Land“ - hier thematisiert Milan Jemini, dass Roma kein eigenes Land haben und durch die Gewaltund Marginalisierungserfahrung im Kosovo bis heute nicht in ihre Herkunftsorte zurückkehren können. Auch Kinder, die in Deutschland geboren wurden, sind marginalisiert aufgrund des prekären Aufenthalts. In der Familie Jemini beispielsweise hat Milan Jemini eine Niederlassungserlaubnis, aber sein in Deutschland geborenes Kind nur eine Duldung. Auch folgende Generationen sind, wie dieser Fall exemplarisch zeigt, häufig marginalisiert aufgrund des prekären Aufenthalts und der damit verbundenen strukturellen Diskriminierung. Gleichzeitig wirken die traumatisierenden Erlebnisse der Gewalt-, Vertreibungs- und Fluchterfahrungen 1999 nach. Milan Jemini deutet aus der Gegenwartsperspektive: „Mir geht das überhaupt nicht aus dem Kopf es bleibt immer da es geht nie weg und kann man auch nicht vergessen Leichen als kleines Kind wenn man so was sieht würde man sich nicht mehr trauen so ich weiß nicht wie ich das sagen soll." 


\section{$4 \quad$ Fazit}

Die empirische Erhebung zeigt, dass je nach Zeitpunkt und Herkunftskontext der Migration bzw. Flucht sowohl unterschiedliche Kriegserfahrungen innerhalb der Familien bestehen als auch unterschiedliche Mobilitätsstrategien. Die Befunde verdeutlichen erstens, wie wenig den nachgeborenen Generationen über die Familienvergangenheit erzählt wurde, zweitens, wie sehr sich die Konstruktionen der kollektiven Zugehörigkeiten in den Generationen unterscheiden, und drittens, wie stark die Familiendynamiken von Migration und transnationalen Verflechtungen bestimmt sind. Es wird deutlich, dass mehrheitlich wenig bis kein Wissen in folgenden Generationen über Erlebnisse der Vorfahren im Zweiten Weltkrieg vorhanden ist. Einen Sonderfall stellt die Familie Markovic dar, in der das Wissen, dass Vorfahren als TitoPartisanen gekämpft hatten, in die Enkelgeneration tradiert wurde, und für eine Heroisierung der eigenen Vorfahren sorgte. Hier wird sichtbar, dass die Phase der sozialen Rehabilitation während der Zeit Tito-Jugoslawiens die gesellschaftliche Etablierung und familiengeschichtliche Memorierung beeinflusste.

Die Kriegs- und Fluchterfahrungen infolge des Zerfalls Jugoslawiens verweisen in den biographisch-narrativen Interviews mit Roma aus dem Kosovo auf primäre Traumatisierungserfahrungen. Konnten die psychisch belastenden Erfahrungen in den Interviews häufig nur fragmentarisch zur Sprache gebracht werden, so wurde dennoch deutlich, dass diese bis in die Gegenwart wirken. Es zeigt sich, dass die Kriegserfahrungen von Roma im Prozess des Zerfalls Jugoslawiens bis heute gesellschaftspolitisch nicht aufgearbeitet wurden und somit auch auf der individualpsychologischen Ebene schwer zu bewältigen sind.

Die Rekonstruktionen der Familien- und Lebensgeschichten verdeutlichen, dass es Dynamiken vielfacher Formen von Mobilität, transnationaler Migration, „, freiwilliger“ Rückkehr oder Abschiebung gibt sowie erneute Migrationen nach Deutschland und auch in die „Herkunftsländer“ zurück. Diese vielfach unfreiwilligen Migrationen, das Gefühl der Unsicherheit sowie restriktives Verhalten seitens der Behörden bedingen psychische Belastungen. So deutet Sarah Petrovic (1968 in Niś geboren) aus der Gegenwartsperspektive ihre Interaktionserfahrungen mit der Ausländerbehörde: „Dann fing er an auf einen Monat zu verlängern dann 15 Tage Terror Terror psychischen Terror mit uns gemacht und wir konnten das nicht mehr uns ist bis hierhin gekommen wir konnten das nicht mehr wir haben ne mein Mann wurde krank ich wurde krank von dem ganzen Stress dieser ganzen Situation ich wurde krank ne wir konnten nicht mehr irgendwann sind wir gegangen wir konnten das nicht mehr." 


\section{Literatur}

Ausländerrecht (2008): Ausländerrecht AuslR. 22. Auflage. München: Deutscher Taschenbuch Verlag.

Baučić, I. (1973): Yugoslavia as a Country of Emigration. In: Options Méditerranéennes, 22, 55-66.

Becker, D. (2014): Die Erfindung des Traumas. Verflochtene Geschichten. Gießen: Psychosozial.

Bhabha, H. K. (2000): Die Verortung der Kultur. Tübingen: Stauffenberg.

Bundesgesetzblatt (2016): Bundesgesetzblatt Jahrgang 2016, Teil I Nr. 12. Online: http://www.bgbl.de/xaver/bgbl/start.xav?startbk=Bundesanzeiger_BGBl\&ju mpTo=bgbl116s0390.pdf <14.04.2020>.

Djuric, R./Becken, J./Bengsch, A. B. (1996): Ohne Heim, ohne Grab. Die Geschichte der Roma und Sinti. Berlin: Aufbau-Verlag.

European Roma Rights Centre (2011): Abandoned Minority. Roma Rights History in Kosovo. Online: www.errc.org/uploads/upload_en/file/abandonedminority-roma-rights-history-in-kosovo-dec-2011.pdf <05.05.2020>.

Fings, K./Lissner, C./Sparing, F. (1992): ...einziges Land, in dem Judenfrage und Zigeunerfrage gelöst. Die Verfolgung der Roma im faschistisch besetzten Jugoslawien 1941-1945. Köln: Rom e.V.

Goeke, P. (2007): Transnationale Migrationen. Post-jugoslawische Biografien in der Weltgesellschaft. Bielefeld: transcript.

Gesellschaft für bedrohte Völker (Hrsg.) (2018): Anzeichen von Langzeitschäden bei Roma aus verseuchten UN-Flüchtlingslagern mehren sich! Online: http://www.gfbv.de/fileadmin/redaktion/Reporte_Memoranden/2018/Koso vo_Langzeitschaeden_bei_Bleivergiftung_Report_GfbV.pdf $<05.05 .2020>$.

Hancock, I. (2002): We Are the Romani People. Ame sam e Rromane džene. Hertfordshire: University of Hertfordshire Press.

Keilson, H. (1979): Sequentielle Traumatisierung bei Kindern. Deskriptiv-klinische und quantifizierend-statistische follow-up Untersuchung zum Schicksal der jüdischen Kriegswaisen in den Niederlanden. Stuttgart: Ferdinand Enke.

Lawler, A. (2017): Whose Memorial? The Arapova Dolina Monument in Leskovac, Serbia. In: Roma and Conflict: Understanding the Impact of War and Political Violence. Roma Rights Journal of the European Roma Rights Center, 1, 1722.

Morokvašić, M. (1987): Jugoslawische Frauen. Die Emigration und danach. Frankfurt a. M./Basel: Stroemfeld/Roter Stern. 
Nederveen Pieterse, J. (2005): Hybridität, na und? In: Allolio Näcke, L./Kalscheuer, B./Manzeschke, A. (Hrsg.): Differenzen anders denken. Bausteine zu einer Kulturtheorie der Transdifferenz. Frankfurt a. M./New York: Campus, 396-430.

Pisarri, M. (2014): The Suffering of the Roma in Serbia during the Holocaust. Belgrad: Forum for Applied History.

Radenbach, N./Rosenthal, G. (2012): Das Vergangene ist auch Gegenwart, das Gesellschaftliche ist auch individuell. Zur Notwendigkeit der Analyse biographischer und historischer ,Rahmendaten'. In: Sozialer Sinn, 13(1), 3-37.

Rosa Luxemburg Stiftung Südosteuropa (Hrsg.) (2016): Von wegen sicher. Das Konzept der sicheren Herkunftsstaaten in der Kritik. Berlin. Online: www.rosalux.de/publikation/id/9232/von-wegen-sicher-1/ <05.05.20>.

Rosenthal, G. (1995): Erlebte und erzählte Lebensgeschichte. Gestalt und Struktur biographischer Selbstbeschreibungen. Frankfurt a. M./New York: Campus.

Rosenthal, G. (Hrsg.) (1997): Der Holocaust im Leben von drei Generationen. Familien von Überlebenden der Shoah und von Nazi-Tätern. Gießen: Psychosozial-Verlag.

Rosenthal, G. (2001): Transgenerationale Folgen von Verfolgung und Täterschaft. In: Streeck-Fischer, A./Sachsse, U./Ökzan, I. (Hrsg.): Körper Seele Trauma. Biologie, Klinik und Praxis. Göttingen: Vandenhoeck \& Ruprecht, 174-206.

Rosenthal, G. (2005): Interpretative Sozialforschung. Eine Einführung. Weinheim/München: Juventa.

Schütze, F. (1977): Die Technik des narrativen Interviews in Interaktionsfeldstudien. Arbeitsbereiche und Forschungsmaterialien Nr. 1 der Universität Bielefeld. Fakultät für Soziologie.

Schütze, F. (1983): Biographieforschung und narratives Interview. In: Neue Praxis, 3, 283-294.

Sichelschmidt, D. (2015): Wie meine Verfolgung begann. DIE ZEIT Nr. 40/2015, 1. Oktober 2015. Online: https://www.zeit.de/2015/40/roma-jugoslawienbuergerkrieg-verfolgung?sort $=\mathrm{desc}<19.05 .2020>$.

Sundhausen, H. (1999): Bevölkerungsentwicklung und Sozialstruktur. In: Hatschikjan, M./Troebst, S. (Hrsg.): Südosteuropa. Gesellschaft, Politik, Wirtschaft, Kultur. München: Beck, 136-150.

Sundhausen, H. (2014): Jugoslawien und seine Nachfolgestaaten 1943-2011. Eine ungewöhnliche Geschichte des Gewöhnlichen. 2. durchgesehene Auflage. Wien/Köln/Weimar: Böhlau. 
Vojak, D. (2017): Roma Also Fought. The History of Romani Participation in the Anti-Fascist Movement in Croatia during World War II. In: Roma and Conflict. Understanding the Impact of War and Political Violence. Roma Rights Journal of the European Roma Rights Center, 1, 9-16.

Vukovic, J. (2016): Warum Serbien kein sicheres Herkunftsland für Roma ist. In: Rosa Luxemburg Stiftung Südosteuropa (Hg.): Von wegen sicher. Das Konzept der sicheren Herkunftsstaaten in der Kritik. Berlin, 19-25. Online: www.rosalux.de/publikation/id/9232/von-wegen-sicher-1/ <05.05.2020>.

Zeidler, K. (2017): Prosecuting War Crimes against Roma in the Yugoslav Wars. The Case of Skočić (Zvornik V) at the Serbian War Crimes Tribunal. In: Roma and Conflict. Understanding the Impact of War and Political Violence. Roma Rights Journal of the European Roma Rights Center, 1, 41-49. 